Check for updates

The BMJ

Cite this as: $B M J 2021 ; 374: \mathrm{n} 2017$ http://dx.doi.org/10.1136/bmj.n2017 Published: 13 August 2021

\section{Covid-19: One in nine patients in hospital with covid during first wave became infected after admission}

\section{Elisabeth Mahase}

Around one in nine hospital patients who had covid-19 during the first wave of the pandemic in the UK were infected after being admitted, researchers have reported. ${ }^{1}$

The team of researchers from the universities of Lancaster, Liverpool, Edinburgh, Birmingham, and Imperial College London looked at the records of 82 ooo patients with covid-19 in UK hospitals who were enrolled in the International Severe Acute Respiratory and emerging Infections Consortium (ISARIC) Clinical Characterisation Protocol UK study ${ }^{2}$ and who became ill before 1 August 2020.

The results, published as correspondence in the Lancet, showed that $11.3 \%$ (95\% confidence interval $11.1 \%$ to $11.6 \%$ ) of patients with covid-19 in 314 hospitals became infected after admission between February and August 2020. This peaked at more than $15.8 \%$ (17.6\% (15.8 to 19.6)) of patients by the middle of May 2020 .

The researchers said there were notable differences between settings, with hospitals providing acute and general care reporting lower proportions of hospital acquired infections (9.7\%) than residential community care hospitals (61.9\%) and mental health hospitals (67.5\%).

Chris Green, consultant physician in infectious diseases and a coauthor of the letter, said in a press release it was likely that several factors contributed to the rate of hospital infection. "These include the large numbers of patients admitted to hospitals with limited facilities for case isolation, limited access to rapid and reliable diagnostic testing in the early stages of the outbreak, the challenges around access to and best use of PPE [personal protective equipment], our understanding of when patients are most infectious in their illness, some misclassification of cases due to presentation with atypical symptoms, and an underappreciation of the role of airborne transmission."

The authors also noted that their figures were likely to be an underestimate of the true number of patients who were infected with SARS-CoV-2 in hospital, because they were not able to identify patients infected during admission but discharged before showing symptoms or patients infected during another healthcare visit before admission.

\section{Improvements since the first wave}

However, the researchers said that rates of hospital infection had fallen since the first wave, so people should not be deterred from attending hospital if they were unwell.

They wrote, "Unlike at the beginning of the pandemic, there are opportunities to pre-empt hospital acquired infections and break chains of transmission through regular patient, resident, and staff testing including point of care diagnostics, as recently introduced for NHS staff, coupled with robust hospital infection prevention and control policies that include staff vaccination, environmental disinfection, and appropriate isolation, all supported by sentinel monitoring systems.”

1 Read JM, Green CA, Harrison EM, etal. Hospital-acquired SARS-CoV-2 infection in the UK's first COVID-19 pandemic wave. Lancet 2021. doi: 10.1016/S0140-6736(21)01786-4.

2 ISARIC4C (Coronavirus Clinical Characterisation Consortium). https://isaric4c.net,

This article is made freely available for use in accordance with BMI's website terms and conditions for the duration of the covid-19 pandemic or until otherwise determined by BMJ. You may use, download and print the article for any lawful, non-commercial purpose (including text and data mining) provided that all copyright notices and trade marks are retained. 